\title{
Amplification de Bragg de la raie Si Ka émise par une multicouche Mo/Si irradiée par un faisceau d'électrons : vers un laser $X$ à rétroaction distribuée ?
}

\author{
P. Jonnard, J.-M. André, C. Bonnelle, F. Bridou ${ }^{1}$ et B. Pardo ${ }^{1}$ \\ Laboratoire de Chimie Physique-Matière et Rayonnement, Université Pierre et Marie Curie, \\ UMR 7614 du CNRS, 11 rue Pierre et Marie Curie, 75231 Paris cedex 05, France \\ ${ }^{1}$ Laboratoire Charles Fabry de l'Institut d'Optique, UMR 8501 du CNRS, bâtiment 503, \\ Centre Scientifique d'Orsay, BP. 147, 91403 Orsay cedex, France
}

\begin{abstract}
Résumé: Nous présentons les modulations d'intensité de la raie Si K $\alpha$ émise par une structure multicouche en fonction de l'angle de sortie des photons. L'observation est réalisée autour de la direction correspondant à la direction de Bragg de la raie Si K $\alpha$ par la multicouche. L'échantillon est irradiée par un faisceau d'électrons dont l'énergie est comprise entre 2 et $6 \mathrm{keV}$. Une importante variation d'intensité est observée dans le domaine angulaire correspondant à la figure de diffraction de la structure émissive. Une augmentation voisine de $15 \%$ de l'intensité de la raie est mesurée dans la direction de Bragg de la multicouche quelle que soit l'énergie des électrons incidents. La modulation est interprétée sur la base du théorème de réciprocité. A la suite de la proposition de Yariv and Yeh [J. Opt. Soc. Am 67 (1977) 438], nous envisageons une application des structures multicouches comme résonateur pour parvenir à un laser $\mathrm{X}$ à rétroaction distribuée.
\end{abstract}

\section{INTRODUCTION}

La théorie dynamique de la diffraction du rayonnement $\mathrm{X}$ prévoit qu'une raie caractéristique $X$ émise par un élément entrant dans la composition d'une structure périodique peut être diffractée dans les conditions de Bragg par cette même structure [1]. Les interférences dues au processus de diffraction provoquent une modulation de l'intensité de la raie en fonction de l'angle de sortie dans un domaine angulaire étroit satisfaisant la condition de Bragg. De telles modulations ont déjà été observées dans les conditions de fluorescence (irradiation par des photons X) [2].

Dans ce travail, nous mesurons l'intensité de la raie Si K $\alpha$ émise par une multicouche périodique sous excitation électronique, en fonction de la direction d'observation. Nous cherchons en particulier à analyser le rôle des conditions d'excitation.

\section{EXPERIENCE}

La multicouche $\mathrm{Mo} / \mathrm{Si}$ est constituée de 40 bicouches $\mathrm{Mo} / \mathrm{Si}$ déposées par évaporation sous faisceau électronique sur un substrat plan et poli de silicium. Les épaisseurs des couches de Mo et Si valent respectivement $1,6 \mathrm{~nm}$ et $3,2 \mathrm{~nm}$ ce qui correspond à une période de $4,8 \mathrm{~nm}$. Un faisceau d'électrons délivré par un canon de Pierce (Kimball Instruments, 0-10 keV, 0$10 \mathrm{~mA}$ ) est utilisé pour ioniser le niveau $1 \mathrm{~s}$ des atomes de silicium présents dans la 
multicouche (énergie d'ionisation : $1840 \mathrm{eV}$ ). Le montage expérimental est tel que les directions des électrons incidents et des photons détectés sont orthogonales. Ainsi l'angle $\alpha$ entre la surface de la multicouche et la direction de sortie des photons détectés est égal à l'angle d'incidence des électrons sur l'échantillon. L'axe de rotation du porte-échantillon est perpendiculaire au plan défini par la direction du faisceau d'électrons et celle des photons $\mathrm{X}$ détectés. Notre porte-échantillon rotatif permet de faire varier l'angle $\alpha$ au voisinage de l'angle de Bragg de la multicouche pour la raie $\mathrm{Si} K \alpha(76.8 \mathrm{mrad})$ avec une précision de $\pm 1,5 \mathrm{mrad}$.

L'émission $\mathrm{Si} K \alpha(\lambda=0,713 \mathrm{~nm})$ est analysée avec un spectromètre $\mathrm{X}$ à très haute résolution [3] utilisant comme élément dispersif un cristal $\mathrm{InSb}$ (111) travaillant au premier ordre. L'angle d'acceptance du spectromètre est égal à $0,4 \mathrm{mrad}$. Pour notre expérience, le spectromètre est utilisé comme monochromateur et il est positionné pour détecter la valeur pic de l'intensité de l'émission Si K $\alpha$; celle-ci est mesurée en fonction de l'angle $\alpha$. Les mesures sont effectuées pour trois valeurs de l'énergie des électrons : 2,1, 4 et $6 \mathrm{keV}$.

\section{RESULTATS}

Sur la figure 1, nous avons tracé les variations de l'intensité en fonction de $\alpha$ entre 35 et $105 \mathrm{mrad}$ pour les trois valeurs de l'énergie des électrons. Ces courbes présentent un maximum pour un même angle $\alpha$ pris comme origine (zéro) dans la figure 1. Cet angle est très voisin de l'angle de Bragg.

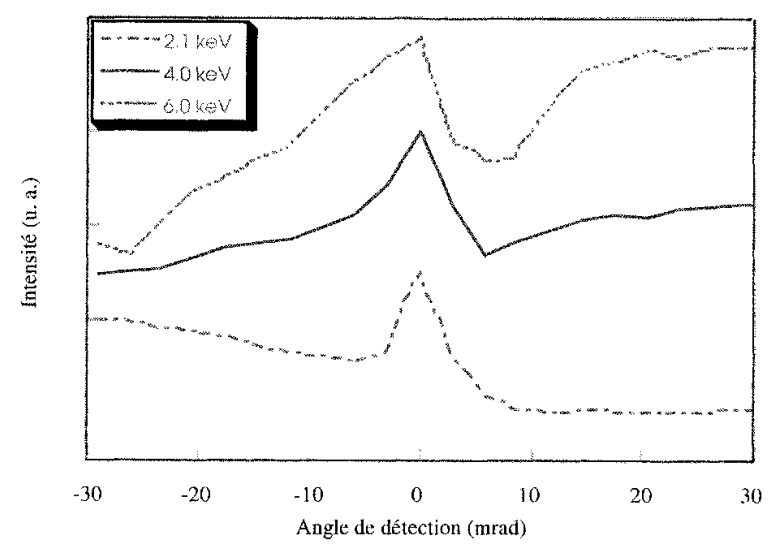

Figure 1 : Intensité de l'émission Si K $\alpha$ de la multicouches Mo/Si en fonction de l'angle de sortie $\alpha: 2.1 \sim \mathrm{keV}$ (ligne continue-tirets); 4 keV (ligne continue); $6 \sim \mathrm{keV}$ (tirets).

A 4 et $6 \mathrm{keV}$, un "creux" apparaît du côté des grands angles par rapport à ce maximum. A $2,1 \mathrm{keV}$ ce creux est très peu prononcé. L'augmentation d'intensité au niveau du pic est estimée à environ $15 \%$ dans les trois cas. Des expériences similaires effectuées avec un échantillon massif de silicium ne montrent pas de modulation de l'intensité dans le domaine angulaire exploré (variation monotone), ce qui prouve que les modulations observées dans le cas de la multicouche sont liées au caractère périodique de la structure multicouche. Les modulations observées s'étendent sur $30 \mathrm{mrad}$. A l'aide d'un modèle optique, nous avons calculé la largeur angulaire de la courbe de réflexion de la multicouche à la longueur d'onde correspondant à l'émission Si-K $\alpha$; celle-ci est du même ordre de grandeur que le domaine où 
nous avons observé les modulations ; autrement dit, les modulations s'étendent sur l'ensemble du domaine de Bragg concerné.

\section{COMPARAISON AVEC LA THEORIE}

Les variations angulaires ont été calculées à partir d'un modèle similaire à celui utilisé précédemment pour traiter la diffraction de Bragg par une multicouche d'une émission caractéristique induite par des photons $X$ (fluorescence) [2] ; le modèle repose sur le théorème de réciprocité [4] et l'emploi du formalisme matriciel pour traiter la propagation du rayonnement $X$ dans la multicouche [5]. Dans le cas présent nous faisons l'approximation grossière que le taux d'ionisation est indépendant de la profondeur, c'est-à-dire que l'on néglige le ralentissement des électrons. La simulation de la variation d'intensité en fonction de l'angle $\alpha$ est portée sur la figure 2, et est comparée à la variation expérimentale à $4 \mathrm{keV}$. Dans cette figure, l'angle zéro correspond à l'angle de Bragg de la courbe théorique ; les courbes expérimentale et théorique sont ajustées à l'angle correspondant à leur saut d'intensité. L'accord entre les deux courbes est plutôt satisfaisant dans la région des modulations. Pour 2,1 et $6 \mathrm{keV}$, l'accord entre expérience et théorie est moins bon. La source principale de désaccord doit probablement être attribuée à notre approximation consistant à supposer une distribution homogène du taux d'ionisation à l'intérieur de la multicouche. En utilisant un modèle semi-empirique [6,7], nous estimons que les ionisations du niveau $\mathrm{K}$ se produit dans les 4 premières bicouches à $2,1 \mathrm{keV}$, dans presque toute la structure multicouche à $4 \mathrm{keV}$ et aussi dans une partie du substrat à $6 \mathrm{keV}$. Ainsi à $4 \mathrm{keV}$, la distribution spatiale des ionisations coïncide dans l'expérience et la théorie, ce qui peut expliquer un meilleur accord par rapport au cas 2,1 et $6 \mathrm{keV}$.

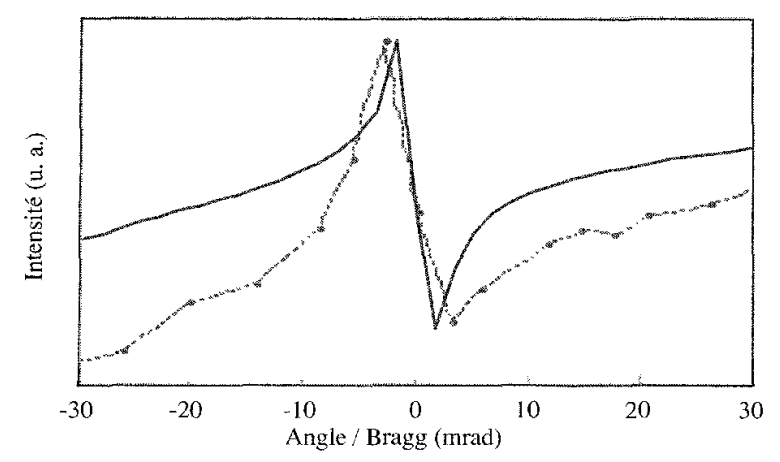

Figure 2 : Intensité de l'émission $\mathrm{Si} K \alpha$ de la multicouches $\mathrm{Mo} / \mathrm{Si}$ en fonction de l'angle de sortie $\alpha$ : expérience à 4 -keV (ligne continue-points); simulation (ligne continue).

\section{CONCLUSION}

Nous avons montré qu'il était possible d'augmenter l'intensité de l'émission d'une raie caractéristique émise au sein d'une structure multicouche périodique dans la direction satisfaisant à la condition de Bragg, l'ionisation étant induite par un faisceau d'électrons. En modifiant l'énergie des électrons excitateurs, on observe une modification des variations 
angulaires de l'intensité en fonction de l'angle $\alpha$. Cependant l'augmentation d'intensité demeure la même quelle que soit l'énergie des électrons, au moins dans le cas abordé dans cette étude.

Notre travail prouve qu'il est possible d'utiliser simultanément une structure multicouche périodique comme source de rayonnement $\mathrm{X}$ caractéristique et comme résonateur pour cette émission. Il s'agit donc d'une première étape dans la réalisation d'un laser X à rétroaction distribuée tel que l'ont proposée Yariv [8] et de Yariv and Yeh [9]. Une amplification conduisant à une émission stimulée pourrait se produire si, dans un système multicouche semblable à celui utilisé dans ce travail, l'un des matériaux constiuant un type de couches présentait un gain pour l'émission considérée.

\section{Références}

[1] James R. W. , The Optical Principles of the Diffraction of X-rays (G. Bell and Sons, London, 1982) pp.438-456.

[2] Chauvineau J.-P., Bridou F., J. Phys IV (Fr) 6 (1996) C7-53.

[3] Bonnelle C. et al., Rev. Sci. Instrum. 65 (1994) 3466.

[4] Lorentz H. A., Proc. Amsterdam Acad. 8 (1905) 401.

[5] Pardo B., Mégadémini T., André J.-M., Rev. Phys. Appl. 23 (1988) 1579.

[6] P.-F. Staub, X-Ray Spectrom. 27 (1998) 43.

[7] P.-F. Staub et al., X-Ray Spectrom. 27 (1998) 58.

[8] A. Yariv, Appl. Phys. Lett. 25 (1974) 105.

[9] A. Yariv et P. Yeh, J. Opt. Soc. Am. 67 (1997) 438. 
Sources, optique et applications VUV 